\title{
Derivation and applications of inequalities of Ostrowski type for n-times differentiable mappings for cumulative distribution function and some quadrature rules
}

\author{
Ather Qayyum ${ }^{a, *}$, Abdul Rehman Kashif ${ }^{b}$, Muhammad Shoaib $^{c}$, Ibrahima Faye ${ }^{a}$ \\ ${ }^{a}$ Department of Fundamental and Applied Sciences, Universiti Teknologi PETRONAS, 32610 Bandar Seri Iskandar, Perak Darul \\ Ridzuan, Malaysia. \\ ${ }^{b}$ Department of Mathematics, University of Hail, 2440, Saudi Arabia. \\ ${ }^{c}$ Abu Dhabi Mens College, Higher Colleges of Technology, P.O. Box 25035, Abu Dhabi, United Arab Emirates.
}

Communicated by M. Bohner

\begin{abstract}
In this paper new integral inequalities of Ostrowski type are developed for n-times differentiable mappings. Some well known inequalities become special cases of the inequalities obtained in this paper. With the help of obtained inequalities, we will derive new and efficient quadrature rules which are analyzed with the help of specific examples. We also give applications for cumulative distribution function. (c)2016 All rights reserved.
\end{abstract}

Keywords: Ostrowski inequality, numerical integration, composite quadrature rule, cumulative distributive function.

2010 MSC: 26D15, 26D20, 26D99.

\section{Introduction}

In 1938, Ostrowski [14] obtained an integral inequality:

\footnotetext{
${ }^{*}$ Corresponding author

Email addresses: atherqayyum@gmail.com (Ather Qayyum), kashmology@gmail.com (Abdul Rehman Kashif), safridi@gmail.com (Muhammad Shoaib), ibrahima_faye@petronas.com.my (Ibrahima Faye)
} 
Theorem 1.1. Let $f:[a, b] \rightarrow \mathbb{R}$ be continuous on $[a, b]$ and differentiable on $(a, b)$, whose derivative is bounded on $(a, b)$, then

$$
|S(f ; a, b)| \leq\left[\left(\frac{b-a}{2}\right)^{2}+\left(x-\frac{a+b}{2}\right)^{2}\right] \frac{M}{b-a}
$$

for all $x \in[a, b]$, where the functional $S(f ; a, b)$ represent the deviation of $f(x)$ from its integral mean over $[a, b]$,

$$
S(f ; a, b)=f(x)-M(f ; a, b)
$$

and

$$
M(f ; a, b)=\frac{1}{b-a} \int_{a}^{b} f(x) d x
$$

is the integral mean.

If we assume that $f^{\prime} \in L_{\infty}[a, b]$ and $\left\|f^{\prime}\right\|_{\infty}=\underset{t \in[a, b]}{e s s}\left|f^{\prime}(t)\right|$ then $M$ in 1.1 may be replaced by $\left\|f^{\prime}\right\|_{\infty}$.

The inequality of Ostrowski type is considered the most useful inequality in mathematical analysis. Some of the classical inequalities for means can be derived from [2] for particular choices of the function $f$. Many researchers have given considerable attention to the inequality (1.1) and its various generalizations, have appeared in the literature, to mention a few, see [1, 4, 5, 6, 8, 9, 10, 11, 13, 16, 20, 21, and the references cited therein.

In the recent year, many new inequalities similar to (1.1) have been established; see [7, 15, 17, 18, 19]. In this current work we will extend, subsume and generalize Ostrowski's inequality for n-times differentiable mappings by using a more generalized kernel. The generalized kernel is expected to reduce approximation errors.

\section{Main Results}

The following lemma is required to prove the main theorem.

Lemma 2.1. Let $f:[a, b] \rightarrow \mathbb{R}$ be an n-time differentiable function such that $f^{(n-1)}(x)$ for $n \in N$ is absolutely continuous on $[a, b]$, then we have the identity

$$
\begin{aligned}
\int_{a}^{b} P_{n}(x, t) f^{(n)}(t) d t= & (-1)^{n+1} \sum_{k=0}^{n-1} \frac{1}{(k+1) !}\left[\left((-1)^{k} A(x-a)^{k+1}+B(b-x)^{k+1}\right) f^{(k)}(x)\right. \\
& \left.+(-1)^{n} A \int_{a}^{x} f(t) d t+B \int_{x}^{b} f(t) d t\right]
\end{aligned}
$$

for all $x \in[a, b]$, where $\alpha, \beta \in \mathbb{R}$ are non negative and not both zero. Consider $P(x,):.[a, b] \rightarrow \mathbb{R}$, the peano kernel

$$
P_{n}(x, t)=\left\{\begin{array}{c}
\frac{A}{n !}(t-a)^{n}, a \leq t \leq x, \\
\frac{B}{n !}(t-b)^{n}, x<t \leq b,
\end{array}\right.
$$

where,

$$
\begin{aligned}
& A=\frac{\alpha}{(\alpha+\beta)(x-a)}, \\
& B=\frac{\beta}{(\alpha+\beta)(b-x)},
\end{aligned}
$$

$n \geq 1, k \geq 1$ are natural numbers and $f^{(0)}(x)=f(x)$. 
Proof. The proof of 2.1) is established using mathematical induction.

Take $n=1$,

$$
\text { L.H.S of } 2.1=\int_{a}^{b} P_{1}(x, t) f^{\prime}(t) d t .
$$

After integration by parts, we get

$$
\int_{a}^{b} P_{1}(x, t) f^{\prime}(t) d t=f(x)-\frac{1}{\alpha+\beta}\left[\frac{\alpha}{x-a} \int_{a}^{x} f(t) d t+\frac{\beta}{b-x} \int_{x}^{b} f(t) d t\right] .
$$

Equation (2.3) is identical to the R.H.S of (2.1) for $n=1$.

Assume that (2.1) is true for $n$ and let us prove it for $n+1$. That is, we have to prove the equality

$$
\begin{aligned}
\int_{a}^{b} P_{n+1}(x, t) f^{(n+1)}(t) d t= & (-1)^{n+2} \sum_{k=0}^{n} \frac{1}{(k+1) !}\left[(-1)^{k} A(x-a)^{k+1}+B(b-x)^{k+1}\right] f^{(k)}(x) \\
& +(-1)^{n+1}\left[A \int_{a}^{x} f(t) d t+B \int_{x}^{b} f(t) d t\right]
\end{aligned}
$$

where

$$
P_{n+1}(x, t)=\left\{\begin{array}{l}
\frac{A}{(n+1) !}(t-a)^{n+1}, a \leq t \leq x, \\
\frac{B}{(n+1) !}(t-b)^{n+1}, x<t \leq b .
\end{array}\right.
$$

We integrate the left hand side of 2.4

$$
\begin{aligned}
\int_{a}^{b} P_{n+1}(x, t) f^{(n+1)}(t) d t= & \frac{1}{(n+1) !}\left[A \int_{a}^{x}(t-a)^{n+1} f^{(n+1)}(t) d t+B \int_{x}^{b}(t-b)^{n+1} f^{(n+1)}(t) d t\right] \\
= & \frac{1}{(n+1) !}\left[A(x-a)^{n+1}+B(-1)(x-b)^{n+1}\right] f^{(n)}(x) \\
& -\frac{(n+1)}{(n+1) !}\left[A \int_{a}^{x}(t-a)^{n} f^{(n)}(t) d t+B \int_{x}^{b}(t-b)^{n} f^{(n)}(t) d t\right] \\
= & \frac{1}{(n+1) !}\left[A(x-a)^{n+1}+B(-1)^{n+2}(b-x)^{n+1}\right] f^{(n)}(x) \\
& -\frac{1}{n !}\left[A \int_{a}^{x}(t-a)^{n} f^{(n)}(t) d t+B \int_{x}^{b}(t-b)^{n} f^{(n)}(t) d t\right] .
\end{aligned}
$$

Multiplying both sides with $(-1)^{n}$, we get

$$
\begin{aligned}
(-1)^{n} \int_{a}^{b} P_{n+1}(x, t) f^{(n+1)}(t) d t= & \frac{1}{(n+1) !}\left[A(-1)^{n}(x-a)^{n+1}+B(b-x)^{n+1}\right] f^{(n)}(x) \\
& -(-1)^{n} \int_{a}^{b} P_{n}(x, t) f^{(n)}(t) d t
\end{aligned}
$$




$$
\begin{aligned}
= & \frac{1}{(n+1) !}\left[A(-1)^{n}(x-a)^{n+1}+B(b-x)^{n+1}\right] f^{(n)}(x) \\
& +\sum_{k=0}^{n-1}\left[\frac{B(b-x)^{k+1}+(-1)^{k} A(x-a)^{k+1}}{(k+1) !}\right] f^{(k)}(x) \\
& -A \int_{a}^{x} f(t) d t-B \int_{x}^{b} f(t) d t \\
= & \sum_{k=0}^{n} \frac{1}{(k+1) !}\left[(-1)^{k} A(x-a)^{k+1}+B(b-x)^{k+1}\right] f^{(k)}(x) \\
& -A \int_{a}^{x} f(t) d t-B \int_{x}^{b} f(t) d t .
\end{aligned}
$$

So,

$$
\begin{aligned}
\int_{a}^{b} P_{n+1}(x, t) f^{(n+1)}(t) d t= & (-1)^{n+2} \sum_{k=0}^{n} \frac{1}{(k+1) !}\left[(-1)^{k} A(x-a)^{k+1}+B(b-x)^{k+1}\right] f^{(k)}(x) \\
& +(-1)^{n+1}\left[A \int_{a}^{x} f(t) d t+B \int_{x}^{b} f(t) d t\right] .
\end{aligned}
$$

Hence 2.4 is proved.

Theorem 2.2. Let $f:[a, b] \rightarrow \mathbb{R}$ be an $n$-time differentiable function such that $f^{(n-1)}(x)$ for $n \in N$ is absolutely continuous on $[a, b]$. Using (2.1), we define

$$
\begin{aligned}
\tau(x ; \alpha, \beta)= & (-1)^{n+1} \sum_{k=0}^{n-1} \frac{1}{(k+1) !}\left[(-1)^{k} A(x-a)^{k+1}+B(b-x)^{k+1}\right] f^{(k)}(x) \\
& +\frac{(-1)^{n}}{\alpha+\beta}[\alpha(M(a, x))+\beta M(x, b)],
\end{aligned}
$$

where $M(f ; a ; b)$ is the integral mean as defined by $(1.3)$, then

$$
|\tau(x ; \alpha, \beta)| \leq\left\{\begin{array}{l}
{\left[A(x-a)^{n+1}+B(b-x)^{n+1}\right] \frac{\left\|f^{(n)}\right\|_{\infty}}{(n+1) !}, f^{(n)} \in L_{\infty}[a, b],} \\
{\left[A^{q}(x-a)^{n q+1}+B^{q}(b-x)^{n q+1}\right]^{\frac{1}{q}} \frac{\left\|f^{(n)}\right\|_{p}}{n !(n q+1)^{\frac{1}{q}}},} \\
f^{(n)} \in L_{p}[a, b], p>1, \frac{1}{p}+\frac{1}{q}=1, \\
{\left[A(x-a)^{n}+B(b-x)^{n}+\left|A(x-a)^{n}-B(b-x)^{n}\right|\right] \frac{\left\|f^{(n)}\right\|_{1}}{2 n !},} \\
f^{(n)} \in L_{1}[a, b] .
\end{array}\right.
$$

Proof. Taking the modulus of (2.1) and using (1.3), we have from 2.5

$$
|\tau(x ; \alpha, \beta)|=\left|\int_{a}^{b} P_{n}(x, t) f^{(n)}(t) d t\right| \leq \int_{a}^{b}\left|P_{n}(x, t)\right| d t \int_{a}^{b}\left|f^{(n)}(t)\right| d t \leq\left\|f^{(n)}(t)\right\|_{\infty} \int_{a}^{b}\left|P_{n}(x, t)\right| d t .
$$


Using (2.2), we get

$$
\int_{a}^{b}\left|P_{n}(x, t)\right| d t=\frac{1}{(n+1) !}\left[A(x-a)^{n+1}+B(b-x)^{n+1}\right] .
$$

Using (2.8) in (2.7), we get first inequality of (2.6).

Using Hölder's integral inequality, from 2.7

$$
|\tau(x ; \alpha, \beta)| \leq\left(\int_{a}^{b}\left|P_{n}(x, t)\right|^{q} d t\right)^{\frac{1}{q}}\left\|f^{(n)}\right\|_{p} .
$$

Now

$$
(\alpha+\beta)\left(\int_{a}^{b}\left|P_{n}(x, t)\right|^{q} d t\right)^{\frac{1}{q}}=\left[\frac{1}{n !(n q+1)}\left(\frac{\alpha}{x-a}\right)^{q}(x-a)^{n q+1}+\frac{1}{n !(n q+1)}\left(\frac{\beta}{b-x}\right)^{q}(b-x)^{n q+1}\right]^{\frac{1}{q}}
$$

This gives

$$
|\tau(x ; \alpha, \beta)| \leq \frac{\left\|f^{(n)}\right\|_{p}}{n !(n q+1)^{\frac{1}{q}}}\left[A^{q}(x-a)^{n q+1}+B^{q}(b-x)^{n q+1}\right]^{\frac{1}{q}} .
$$

This completes the proof of second inequality.

Finally, by using the definition of $\|\cdot\|_{1}-$ norm, we get

$$
|\tau(x ; \alpha, \beta)| \leq \sup _{t \in[a, b]}\left|P_{n}(x, t)\right|\left\|f^{(n)}\right\|_{1},
$$

so

$$
(\alpha+\beta) \sup _{t \in[a, b]}\left|P_{n}(x, t)\right|=\frac{1}{2 n !}\left[\alpha(x-a)^{n-1}+\beta(b-x)^{n-1}\right]+\frac{1}{2 n !}\left|\alpha(x-a)^{n-1}-\beta(b-x)^{n-1}\right| .
$$

Therefore, by using (2.7), we get last inequality. This completes the proof of Theorem 2.2 .

Remark 2.3. If we put $n=1$ and $n=2$ in (2.6), we get the results of Cerone [4] and Qayyum et al [19] respectively.

\section{Perturbed Results using Čebyŝev functional}

In this section, we will establish some new results by using Grüss inequality and the Čebyŝev functional. In 1882, Čebyŝev [3] gave the following inequality.

$$
|T(f, g)| \leq \frac{1}{12}(b-a)^{2}\left\|f^{\prime}\right\|_{\infty}\left\|g^{\prime}\right\|_{\infty},
$$

where $f, g:[a, b] \rightarrow \mathbb{R}$ are absolutely continuous functions, which have bounded first derivatives, and

$$
\begin{aligned}
T(f, g): & =\frac{1}{b-a} \int_{a}^{b} f(x) g(x) d x-\frac{1}{(b-a)^{2}} \int_{a}^{b} f(x) d x \cdot \int_{a}^{b} g(x) d x \\
& =M(f g ; a, b)-M(f ; a, b) M(g ; a, b) .
\end{aligned}
$$


In 1935, Grüss [12] proved the following inequality:

$$
\left|\frac{1}{b-a} \int_{a}^{b} f(x) g(x) d x-\frac{1}{b-a} \int_{a}^{b} f(x) d x \cdot \frac{1}{b-a} \int_{a}^{b} g(x) d x\right| \leq \frac{1}{4}(\Phi-\varphi)(\Gamma-\gamma),
$$

provided that $f$ and $g$ are two integrable functions on $[a, b]$ and satisfy the condition

$$
\varphi \leq f(x) \leq \Phi \text { and } \gamma \leq g(x) \leq \Gamma \text { for all } x \in[a, b] .
$$

The constant $\frac{1}{4}$ is best possible. The perturbed version of the results of Theorem 3.1 can be obtained by using Grüss type results involving the Čebyŝev functional

$$
T(f, g)=M(f g ; a, b)-M(f ; a, b) M(g ; a, b),
$$

where $M$ is the integral mean defined in 1.3$)$.

Theorem 3.1. Let $f:[a, b] \rightarrow \mathbb{R}$ be an $n$-time differentiable function such that $f^{(n-1)}(x)$ for $n \in N$ is absolutely continuous on $[a, b]$ and $\alpha, \beta$ are non-negative real numbers. Then

$$
\begin{aligned}
\left|\tau(x ; \alpha, \beta)-\frac{1}{(\alpha+\beta)}\left[\alpha(x-a)^{n}+\beta(x-b)^{n}\right] \frac{\kappa}{(n+1) !}\right| & \leq(b-a) N(x)\left[\frac{1}{b-a}\left\|f^{(n)}\right\|_{2}^{2}-\kappa^{2}\right]^{\frac{1}{2}} \\
& \leq \frac{(b-a)}{2(\alpha+\beta)}(\Phi-\varphi)(\Gamma-\gamma),
\end{aligned}
$$

where $\tau(x ; \alpha, \beta)$ is as given by 2.5),

$$
\begin{aligned}
& \kappa:=\frac{f^{(n-1)}(b)-f^{(n-1)}(a)}{b-a}, \\
& N^{2}(x):= {\left[\left(\frac{\alpha}{\alpha+\beta}\right)^{2} \frac{(x-a)^{2 n-1}}{(n !)^{2}(2 n+1)}-\left(\frac{\beta}{\alpha+\beta}\right)^{2} \frac{1}{(2 n+1)(n !)^{2}}(x-b)^{2 n-1}\right] \times \frac{1}{b-a} } \\
&-\left(\frac{1}{(b-a)(\alpha+\beta)(n+1) !}\left[\alpha(x-a)^{n}+\beta(x-b)^{n}\right]\right)^{2},
\end{aligned}
$$

and

$$
\begin{aligned}
& \Phi=\sup _{t \in[a, b]} P_{n}(x, t)=\frac{1}{(\alpha+\beta) n !}\left[\alpha(x-a)^{n-1}+\beta(x-b)^{n-1}\right] \\
& \varphi=\inf _{t \in[a, b]} P_{n}(x, t)=0
\end{aligned}
$$

if $n$ is even

$$
\Phi=\sup _{t \in[a, b]} P_{n}(x, t)=\frac{1}{(\alpha+\beta) n !} \alpha(x-a)^{n-1}
$$

and

$$
\varphi=\inf _{t \in[a, b]} P_{n}(x, t)=\frac{-\beta(b-x)^{n-1}}{(\alpha+\beta) n !},
$$

if $n$ is odd. 
Proof. Associating $f(t)$ with $P_{n}(x, t)$ and $g(t)$ with $f^{(n)}(t)$, we obtain

$$
T\left(P_{n}(x, .), f^{(n)}(.) ; a, b\right)=M\left(P_{n}(x, .) f^{(n)}(.) ; a, b\right)-M\left(P_{n}(x, .) ; a, b\right) M\left(f^{(n)}(.) ; a, b\right) .
$$

Now using identity (2.1),

$$
(b-a) T\left(P_{n}(x, .), f^{(n)}(.) ; a, b\right)=\tau(x ; \alpha, \beta)-(b-a) M\left(P_{n}(x, .) ; a, b\right) \kappa,
$$

where $\kappa$ is the secant slope of $f^{\prime}$ over $[a, b]$ as given in (3.6). Now, from 2.1) and (3.2),

$$
\begin{aligned}
(b-a) M\left(P_{n}(x, .) ; a, b\right) & =\int_{a}^{b} P_{n}(x, t) d t \\
& =\frac{1}{(\alpha+\beta) n !}\left[\frac{\alpha}{x-a} \int_{a}^{x}(t-a)^{n} d t+\frac{\beta}{b-x} \int_{x}^{b}(t-b)^{n}\right] d t \\
& =\frac{1}{(\alpha+\beta)(n+1) !}\left[\alpha(x-a)^{n}+\beta(x-b)^{n}\right] .
\end{aligned}
$$

Now combining (3.9) with (3.7), the left hand side of (3.5) is obtained.

Let $f, g:[a, b] \rightarrow \mathbb{R}$ and $f g:[a, b] \rightarrow \mathbb{R}$ be integrable on $[a, b]$, then [4]

$$
\begin{aligned}
|T(f, g)| & \leq T^{\frac{1}{2}}(f, f) T^{\frac{1}{2}}(g, g) & & \left(f, g \in L_{2}[a, b]\right) \\
& \leq \frac{(\Gamma-\gamma)}{2} T^{\frac{1}{2}}(f, f) & & (\gamma \leq g(x) \leq \Gamma, t \in[a, b]) \\
& \leq \frac{1}{4}(\Phi-\varphi)(\Gamma-\gamma) & & (\varphi \leq f(x) \leq \Phi, t \in[a, b]) .
\end{aligned}
$$

Also, note that

$$
\begin{aligned}
0 \leq T^{\frac{1}{2}}\left(f^{(n)}(.), f^{(n)}(.)\right) & =\left[M\left(f^{(n)}(.)^{2} ; a, b\right)-M^{2}\left(f^{(n)}(.) ; a, b\right)\right]^{\frac{1}{2}} \\
& =\left[\frac{1}{b-a} \int_{a}^{b}\left|f^{(n)}(t)\right|^{2} d t-\left(\frac{\int_{a}^{b} f^{(n)}(t) d t}{b-a}\right)^{2}\right]^{\frac{1}{2}} \\
& =\left[\frac{1}{b-a}\left\|f^{(n)}\right\|_{2}^{2}-\kappa^{2}\right]^{\frac{1}{2}} \\
& \leq \frac{(\Gamma-\gamma)}{2},
\end{aligned}
$$

where $\gamma \leq f^{\prime \prime}(t) \leq \Gamma, t \in[a, b]$. Now, for the bounds on 3.8 , we have to determine $T^{\frac{1}{2}}\left(P_{n}(x,),. P_{n}(x,).\right)$, and $\phi$ and $\Phi$ such that $\varphi \leq P_{n}(x,.) \leq \Phi$.

Now from 2.2 , the definition of $P_{n}(x, t)$, we have

$$
T\left(P_{n}(x, .), P_{n}(x, .)\right)=M\left(P^{2}(x, .) ; a, b\right)-M^{2}\left(P_{n}(x, .) ; a, b\right) .
$$

From 3.9 we obtain

$$
M\left(P_{n}(x, .) ; a, b\right)=\frac{1}{(b-a)(\alpha+\beta)(n+1) !}\left[\alpha(x-a)^{n}+\beta(x-b)^{n}\right]
$$


and

$$
\begin{aligned}
(b-a) M\left(P^{2}(x, .) ; a, b\right) & =\left(\frac{\alpha}{\alpha+\beta}\right)^{2} \frac{1}{(n !(x-a))^{2}} \int_{a}^{x}(t-a)^{2 n} d t+\left(\frac{\beta}{\alpha+\beta}\right)^{2} \frac{1}{(n !(b-x))^{2}} \int_{x}^{b}(t-b)^{2 n} d t \\
& =\left(\frac{\alpha}{\alpha+\beta}\right)^{2} \frac{(x-a)^{2 n-1}}{(n !)^{2}(2 n+1)}-\left(\frac{\beta}{\alpha+\beta}\right)^{2} \frac{(x-b)^{2 n-1}}{(2 n+1)(n !)^{2}} .
\end{aligned}
$$

Thus, substituting the above results into 3.12 gives

$$
0 \leq N(x):=T^{\frac{1}{2}}\left(P_{n}(x, .), P_{n}(x, .)\right)
$$

which is given explicitly by (3.7). Combining (3.8), (3.11) and 3.12$)$ gives from the first inequality in $(3.10)$, the first inequality in (3.5). Now utilizing the inequality in (3.11) produces the second result in (3.5). Further, it may be noticed from the definition of $P(x, t)$ in 2.2 that for $\alpha, \beta \geq 0$,

$$
\Phi=\sup _{t \in[a, b]} P_{n}(x, t) \quad \text { and } \varphi=\inf _{t \in[a, b]} P_{n}(x, t) .
$$

Remark 3.2. Similarly, If we put $n=1$ and $n=2$, in Theorem 3.1, we get the results proved in [4] and [19] respectively.

\section{An Application to the Cumulative Distributive Function}

Let $X \in[a, b]$ be a random variable with the cumulative distributive function

$$
F(x)=P_{r}(X \leq x)=\int_{a}^{x} f(u) d u,
$$

where $f$ is the probability density function. In particular,

$$
\int_{a}^{b} f(u) d u=1 .
$$

The following theorem holds.

Theorem 4.1. Let $X$ and $F$ be as above, then

$$
\begin{aligned}
& \left|\sum_{k=0}^{n-1} \frac{(-1)^{n+1}}{(k+1) !}\left[\begin{array}{c}
(-1)^{k} \alpha(x-a)^{k+1}(b-x) \\
+\beta(x-a)(b-x)^{k+1}
\end{array}\right] f^{(k)}(x)+(-1)^{n}[\{\alpha(b-x)-\beta(x-a)\} F(x)-\beta(x-a)]\right| \\
& \quad \leq\left\{\begin{array}{c}
(\alpha+\beta)(b-x)(x-a)\left[A(x-a)^{n+1}+B(b-x)^{n+1}\right] \frac{\left\|f^{(n)}\right\|_{\infty}}{(n+1) !}, f^{(n)} \in L_{\infty}[a, b], \\
(\alpha+\beta)(b-x)(x-a)\left[A^{q}(x-a)^{n q+1}+B^{q}(b-x)^{n q+1}\right]^{\frac{1}{q}} \frac{\left\|f^{(n)}\right\|_{p}}{n !(n q+1)^{\frac{1}{q}}}, f^{(n)} \in L_{p}[a, b], \\
(\alpha+\beta)(b-x)(x-a)\left[\begin{array}{c}
A(x-a)^{n}+B(b-x)^{n} \\
+\left|A(x-a)^{n}-B(b-x)^{n}\right|
\end{array}\right] \frac{\left\|f^{(n)}\right\|_{1}}{2 n !}, f^{(n)} \in L_{1}[a, b] .
\end{array}\right.
\end{aligned}
$$


Proof. From 2.5 and by using the definition of probability density function, we have

$$
\begin{aligned}
\tau(x ; \alpha, \beta)= & \frac{1}{\alpha+\beta} \sum_{k=1}^{n} \frac{(-1)^{n+k}}{k !}\left[\alpha(x-a)^{k-1} f^{(k-1)}(x)+\beta(x-b)^{k-1} f^{(k-1)}(x)\right] \\
& +\frac{(-1)^{n}}{\alpha+\beta}[\alpha(M(a, x))+\beta M(x, b)] \\
= & \frac{1}{\alpha+\beta} \sum_{k=1}^{n} \frac{(-1)^{n+k}}{k !}\left[\alpha(x-a)^{k-1} f^{(k-1)}(x)+\beta(x-b)^{k-1} f^{(k-1)}(x)\right] \\
& +\frac{(-1)^{n}}{\alpha+\beta}\left[\frac{\{\alpha(b-x)-\beta(x-a)\} F(x)}{(x-a)(b-x)}+\frac{\beta}{(b-x)}\right] .
\end{aligned}
$$

Now using (2.6) and (4.2), we get our required result (4.1).

Putting $\alpha=\beta=\frac{1}{2}$ in Theorem 3.1 gives the following result.

Corollary 4.2. Let $X$ be a random variable with the cumulative distributive function $F(x)$ and the probability density function $f(x)$. Then

$$
\begin{aligned}
& \mid \frac{(-1)^{n+1}}{2} \sum_{k=0}^{n-1} \frac{f^{(k)}(x)}{(k+1) !}\left[(-1)^{k}(x-a)^{k+1}(b-x)+(x-a)(b-x)^{k+1}\right] \\
& \quad+(-1)^{n}\left[\left(\frac{a+b}{2}-x\right) 2 F(x)-(x-a)\right] \mid \\
& \leq\left\{\begin{array}{l}
(b-x)(x-a)\left[(x-a)^{n}+(b-x)^{n}\right] \frac{\left\|f^{(n)}\right\|_{\infty}}{2(n+1) !}, \\
(b-x)(x-a)\left[(x-a)^{n q+1-q}+(b-x)^{n q+1-q}\right]^{\frac{1}{q}} \frac{\left\|f^{(n)}\right\|_{p}}{2 n !(n q+1)^{\frac{1}{q}}}, \\
(b-x)(x-a)\left[\begin{array}{c}
(x-a)^{n-1}+(b-x)^{n-1} \\
+\left|(x-a)^{n-1}-B(b-x)^{n-1}\right|
\end{array}\right] \frac{\left\|f^{(n)}\right\|_{1}}{4 n !} .
\end{array}\right.
\end{aligned}
$$

Remark 4.3. The above result allow the approximation of $F(x)$ in terms of $f(x)$. The approximation of

$$
R(x)=1-F(x)
$$

could also be obtained by a simple substitution. $R(x)$ is of importance in reliability theory where $f(x)$ is the probability density function of failure.

Remark 4.4. We put $\beta=0$ in (4.1), assuming that $\alpha \neq 0$ to obtain

$$
\begin{aligned}
\left|(-1)^{n+1} \sum_{k=0}^{n-1} \frac{f^{(k)}(x)}{(k+1) !}\left[(-1)^{k}(x-a)^{k+1}(b-x)\right]+(-1)^{n}(b-x) F(x)\right| \\
\leq\left\{\begin{array}{l}
(b-x)(x-a)^{n+1} \frac{\left\|f^{(n)}\right\|_{\infty}}{(n+1) !}, \\
(b-x)(x-a)\left[(x-a)^{n q+1-q}\right]^{\frac{1}{q}} \frac{\left\|f^{(n)}\right\|_{p}}{n !(n q+1)^{\frac{1}{q}}}, \\
(b-x)(x-a)\left((x-a)^{n-1}+\left|(x-a)^{n-1}\right|\right) \frac{\left\|f^{(n)}\right\|_{1}}{2 n !} .
\end{array}\right.
\end{aligned}
$$

Further we note that

$$
\int_{a}^{b} F(u) d u=\left.u F(u)\right|_{a} ^{b}-\int_{a}^{b} x f(x) d x=b-E(X) .
$$

Remark 4.5. By choosing $n=1$ and $n=2$ in (4.1)-(4.4), we can get results proved in [4] and [19]. 


\section{Some new Ostrowski Type Inequalities for $\alpha=x-a$ and $\beta=b-x$}

Theorem 5.1. Let $f:[a, b] \rightarrow \mathbb{R}$ be differentiable on $(a, b), f^{(n-1)}$ is absolutely continuous on $[a, b]$ and $\gamma \leq f^{(n)}(t) \leq \Gamma \forall t \in[a, b]$, then for all $x \in\left[a, \frac{a+b}{2}\right]$, we have

$$
|X| \leq \frac{Y}{2(b-a)(n+1) !}(S-\gamma)
$$

and

$$
|X| \leq \frac{Y}{2(b-a)(n+1) !}(\Gamma-S)
$$

if $n$ is even and

$$
|X| \leq \frac{Z}{2(b-a)(n+1) !}(S-\gamma)
$$

and

$$
|X| \leq \frac{Z}{2(b-a)(n+1) !}(\Gamma-S)
$$

if $n$ is odd,

where

$$
\begin{aligned}
X:= & (-1)^{n+1} \sum_{k=0}^{n-1} \frac{1}{(b-a)^{2}(k+1) !}\left[(-1)^{k}(x-a)^{k+1}+(b-x)^{k+1}\right] f^{(k)}(x) \\
& +\frac{(-1)^{n}}{(b-a)^{2}} \int_{a}^{b} f(t) d t-\frac{1}{(b-a)^{2}(n+1) !}\left[(x-a)^{n+1}-(x-b)^{n+1}\right] \times f^{(n-1)}(b)-f^{(n-1)}(a), \\
Y:= & (x-a)^{n}[n+1+2(a-x)]+(x-b)^{n}[n+1+2(x-b)]+(n+1)\left[(x-a)^{n}-(x-b)^{n}\right]
\end{aligned}
$$

and

$$
Z:=(x-a)^{n}[n+1+2(a-x)]+2(x-b)^{n+1}+(n+1)(x-a)^{n} .
$$

Proof. From (2.2) and using $\alpha=x-a$ and $\beta=b-x$ and

$$
\frac{1}{b-a} \int_{a}^{b} f^{(n)}(t) d t=\frac{f^{(n-1)}(b)-f^{(n-1)}(a)}{b-a}
$$

and

$$
\frac{1}{b-a} \int_{a}^{b} P_{n}(x, t) d t=\frac{1}{(b-a)(n+1) !}\left[(x-a)^{n+1}-(x-b)^{n+1}\right] \text {, }
$$

we get

$$
\begin{aligned}
\frac{1}{b-a} \int_{a}^{b} P_{n}(x, t) f^{(n)}(t) d t-\frac{1}{(b-a)^{2}} \int_{a}^{b} P_{n}(x, t) d t \int_{a}^{b} f^{(n)}(t) d t \\
=(-1)^{n+1} \sum_{k=0}^{n-1} \frac{1}{(b-a)^{2}(k+1) !}\left[(-1)^{k}(x-a)^{k+1}+(b-x)^{k+1}\right] f^{(k)}(x) \\
\quad+\frac{(-1)^{n}}{(b-a)^{2}} \int_{a}^{b} f(t) d t-\frac{1}{(b-a)^{2}(n+1) !}\left[(x-a)^{n+1}-(x-b)^{n+1}\right] \\
\quad \times f^{(n-1)}(b)-f^{(n-1)}(a) .
\end{aligned}
$$


We suppose that

$$
R_{n}(x)=\frac{1}{b-a} \int_{a}^{b} P_{n}(x, t) f^{(n)}(t) d t-\frac{1}{(b-a)^{2}} \int_{a}^{b} P_{n}(x, t) d t \int_{a}^{b} f^{(n)}(t) d t .
$$

If $C \in R$ is an arbitrary constant, then we have

$$
R_{n}(x)=\frac{1}{b-a} \int_{a}^{b}\left(f^{(n)}(t)-C\right)\left[P_{n}(x, t)-\frac{1}{b-a} \int_{a}^{b} P^{(n)}(x, s) d s\right] d t .
$$

Furthermore, we have

$$
\left|R_{n}(x)\right| \leq \frac{1}{b-a} \max _{t \in[a, b]}\left|P_{n}(x, t)-\frac{1}{b-a} \int_{a}^{b} P^{(n)}(x, s) d s\right| \int_{a}^{b}\left|f^{(n)}(t)-C\right| d t .
$$

Now, if $n$ is even

$$
\begin{aligned}
\mid P_{n}(x, t) & -\frac{1}{b-a} \int_{a}^{b} P^{(n)}(x, s) d s \mid \\
= & \frac{1}{(b-a)(n+1) !} \max \left[\left|\left\{(x-a)^{n}\{n+1-x+a\}+(x-b)^{n+1}\right\}\right|\right. \\
= & \frac{1}{2(b-a)(n+1) !}\left[(x-a)^{n}\{n+1+2(a-x)\}\right. \\
& +(x-b)^{n}\{n+1+2(x-b)\} \\
& \left.+(n+1)\left|\left\{(x-a)^{n}-(x-b)^{n}\right\}\right|\right]
\end{aligned}
$$

and if $n$ is odd

$$
\begin{aligned}
\mid P_{n}(x, t) & -\frac{1}{b-a} \int_{a}^{b} P^{(n)}(x, s) d s \mid \\
\quad= & \frac{1}{(b-a)(n+1) !} \max \left[\left|\left[(x-a)^{n}(n+1-x+a)+(x-b)^{n+1}\right]\right|,\left|(x-b)^{n+1}-(x-a)^{n+1}\right|\right] \\
= & \frac{1}{2(b-a)(n+1) !}\left[(x-a)^{n}[n+1+2(a-x)]+2(x-b)^{n+1}+(n+1)\left|(x-a)^{n}\right|\right] .
\end{aligned}
$$

We also have

$$
\int_{a}^{b}\left|f^{(n)}(t)-\gamma\right| d t=(S-\gamma)(b-a)
$$

and

$$
\int_{a}^{b}\left|f^{(n)}(t)-\Gamma\right| d t=(\Gamma-S)(b-a) .
$$

Therefore, we can obtain (5.1) and (5.4) by using (5.5) to (5.14) taking $C=\gamma$ and $C=\Gamma$ in (5.10), respectively. 


\subsection{Derivation of Numerical Quadrature Rules}

We propose some new quadrature rules involving higher order derivatives of the function $f$. In fact, the following new quadrature rules can be obtained while investigating error bounds using Theorem 5.1 .

$$
\begin{aligned}
Q_{n, 1}(f):= & \int_{a}^{b} f(t) d t \approx \sum_{k=0}^{n-1} \frac{(b-a)^{k+1}}{(k+1) !} f^{(k)}(a)+\frac{(b-a)^{n+1}}{(n+1) !}\left[f^{(n-1)}(b)-f^{(n-1)}(a)\right], \\
Q_{n, 2}(f):= & \int_{a}^{b} f(t) d t \approx \sum_{k=0}^{n-1} \frac{(b-a)^{k+1}}{2^{k+1}(k+1) !}\left[1+(-1)^{k}\right] f^{(k)}\left(\frac{a+b}{2}\right) \\
& +\frac{(b-a)^{n+1}}{2^{n+1}(n+1) !}\left[1+(-1)^{n}\right]\left[f^{(n-1)}(b)-f^{(n-1)}(a)\right] \\
Q_{n, 3}(f):= & \int_{a}^{b} f(t) d t \approx \sum_{k=0}^{n-1} \frac{(b-a)^{k+1}}{4^{k+1}(k+1) !}\left[(-1)^{k}+(3)^{k+1}\right] f^{(k)}\left(\frac{3 a+b}{4}\right) \\
& +\frac{(-1)^{n}(b-a)^{n+1}}{4^{n+1}(n+1) !}\left[1+(-1)^{n}(3)^{n+1}\right]\left[f^{(n-1)}(b)-f^{(n-1)}(a)\right] .
\end{aligned}
$$

To investigate the efficiency of these quadrature rules we integrate various types of functions including Polynomial, Exponential, Logarithmic. The results of the numerical integrations are given in Table 1 with absolute error and the value of $\mathrm{n}$ used to obtain the mentioned accuracy.

$$
\begin{array}{ll}
f_{1}(x)=x^{2}+x+2, & f_{2}(x)=x \sin x, \\
f_{3}(x)=e^{x} \sin x, & f_{4}(x)=x^{2}+\sin x, \\
f_{5}(x)=e^{x^{2}}, & f_{6}(x)=e^{x} \cos \left(e^{x}-2 x\right), \\
f_{7}(x)=x+\cos x, & f_{8}(x)=\log \left(x^{2}+2\right) \sin \left[\log \left(x^{2}+2\right)\right] .
\end{array}
$$

The errors shown in the Table 1 are the absolute value of the difference of the exact value of the integral and its numerical value. All three quadrature rules show exact value of the integral of $f_{1}$ for $n=2$. For a polynomial of degree $k, n=k+1$ will give exact value of the integral $f_{1}$. Though acceptable error estimates can be obtained for smaller values of $n$. to save computational time.

The integral of $f_{5}, Q_{n, 3}(f)$ give an error of the order of $10^{-5}$ for $n=7$ while the other two quadrature rules give a similar error for $n=13$ and $n=16$. Similarly for all other functions $Q_{n, 3}(f)$ report errors of the order of $10^{-5}$ or $10^{-6}$ for relatively smaller values of $n$ as compared to the other two quadrature rules. Specifically, $Q_{n, 3}(f)$ give an excellent estimate for the integrals of $f_{6}, f_{8}$ and $f_{5}$ at $n=6$ and $n=7$ respectively. In general $Q_{n, 3}(f)$ gave better results as compared to the rest of the quadrature rules for much smaller values of $n$. Therefore we can conjecture that $Q_{n, 3}(f)$ is computationally more efficient both in terms of error approximation, simplicity and time. As a rough estimate we integrated $\log \left(x^{2}+2\right) \sin \left[\log \left(x^{2}+2\right)\right]$ using the builtin algorithms of Mathematica 10.0 which took 26.30 seconds to give its approximate answer. To obtain similar approximation for the integral of $f_{8}, Q_{n, 3}(f)$ took less than a second. The performance of some the quadrature rules can be seen to be poor for the integrals $f_{5}, f_{6}$ and $f_{8}$ where we had to take $n$ around 15, 16 and 18 for $Q_{n, 1}(f)$ to achieve a reasonable approximation error. 
Table 1: Performance of the proposed quadrature rules

\begin{tabular}{|c|c|c|c|c|c|}
\hline Sr. No. & Method & $\bar{c} n: \quad Q_{n, 1}(f)$ & 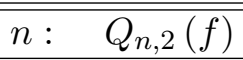 & $\bar{c} n: \quad Q_{n, 3}(f)$ & Exact Value \\
\hline \multirow[t]{2}{*}{1.} & $\int_{0}^{1} f_{1}(x) d x$ & 2.83333 & 2: $\quad 2.83333$ & 2: $\quad 2.83333$ & 2.83333 \\
\hline & Error: & 0 & 0 & 0 & \\
\hline \multirow[t]{2}{*}{2.} & $\int_{0}^{1} f_{2}(x) d x$ & 7: $\quad 0.301101$ & 6: $\quad 0.301149$ & 5: $\quad 0.301153$ & 0.301169 \\
\hline & Error: & 0.00006748 & 0.0000201585 & 0.0000155081 & \\
\hline \multirow[t]{2}{*}{3.} & $\int_{0}^{1} f_{3}(x) d x$ & 8: $\quad 0.909365$ & 6: $\quad 0.909319$ & 5: $\quad 0.909366$ & 0.909331 \\
\hline & Error: & 0.0000348171 & 0.000011471 & 0.0000357479 & \\
\hline \multirow[t]{2}{*}{4.} & $\int_{0}^{1} f_{4}(x) d x$ & 6: $\quad 0.792964$ & 5: $\quad 0.793008$ & 4: $\quad 0.793022$ & 0.793031 \\
\hline & Error: & 0.0000666818 & 0.0000225644 & $8.79 \times 10^{-6}$ & \\
\hline \multirow[t]{2}{*}{5.} & $\int_{0}^{1} f_{5}(x) d x$ & 16: $\quad 1.46271$ & 13: 1.46267 & $7: \quad 1.46257$ & 1.46265 \\
\hline & Error: & 0.0000562577 & 0.000015241 & 0.0000864484 & \\
\hline \multirow[t]{2}{*}{6.} & $\int_{0}^{1} f_{6}(x) d x$ & 18: $\quad 1.31384$ & 12: 1.31385 & 6: $\quad 1.31386$ & 1.31383 \\
\hline & Error & $5.3 \times 10^{-6}$ & 0.0000229733 & 0.0000308985 & \\
\hline \multirow[t]{2}{*}{7.} & $\int_{0}^{1} f_{7}(x) d x$ & 6: $\quad 1.3415$ & 5: $\quad 1.34144$ & 4: $\quad 1.34145$ & 1.34147 \\
\hline & Error: & 0.0000287233 & 0.000027542 & 0.0000160969 & \\
\hline \multirow[t]{2}{*}{8.} & $\int_{0}^{1} f_{8}(x) d x$ & 15: $\quad 0.629715$ & 9: $\quad 0.629866$ & 6: $\quad 0.629677$ & 0.629769 \\
\hline & Error: & 0.0000532107 & 0.0000973014 & 0.0000916031 & \\
\hline
\end{tabular}

\section{References}

[1] R. P. Agarwal, V. Cǔljak, J. Pečarić, Some integral inequalities involving bounded higher order derivatives, Math. Comput. Modelling, 28 (1998), 51-57. 1 .

[2] M. Alomari, M. Darus, Some Ostrowski type inequalities for convex functions with applications to special means, RGMIA Res. Rep. Coll., 13 (2010). 1

[3] P. L. Čebyŝev, Sur less expressions approximatives des integrales definies par les autres prises entre les memes limites, Proc. Math. Soc. Charkov, 2 (1882), 93-98. 3

[4] P. Cerone, A new Ostrowski Type Inequality Involving Integral Means Over End Intervals, Tamkang J. Math., 33 (2002), 109-118. 1, 2.3, 3, 3.2, 4.5

[5] P. Cerone, S. S. Dragomir, J. Roumeliotis, An inequality of Ostrowski type for mappings whose second derivatives are bounded and applications, RGMIA Res. Rep. Coll., 1 (1998). 1

[6] P. Cerone, S. S. Dragomir, J. Roumeliotis, Some Ostrowski type Inequalities for n-time differentiable mappings and applications, Demonstratio Math., 32 (1999), 697-712. 1

[7] S. S. Dragomir, E. Momoniat, A three point quadrature rule for functions of bounded variation and applications, Math. Comput. Modelling, 57 (2013), 612-622. 1

[8] S. S. Dragomir, S. Wang, An inequality of Ostrowski-Grüs type and its applications to the estimation of error bounds for some special means and for some numerical quadrature rules, Comput. Math. Appl., 33 (1997), 15-20. 1

[9] S. S. Dragomir, S. Wang, A new inequality Ostrowski's type in $L_{1}$ - norm and applications to some special means and some numerical quadrature rules, Tamkang J. Math., 28 (1997), 239-244. 1

[10] S. S. Dragomir, S. Wang, A new inequality Ostrowski's type in $L_{p^{-}}$norm, Indian J. Math., 40 (1998), 299-304. 1

[11] S. S. Dragomir, S. Wang, Applications of Ostrowski's inequality to the estimation of error bounds for some special means and to some numerical quadrature rules, Appl. Math. Lett., 11 (1998), 105-109. 1 ] 
[12] G. Grüss, Über das Maximum des absoluten Betrages von $\frac{1}{b-a} \int_{a}^{b} f(x) g(x) d x-\frac{1}{(b-a)^{2}} \int_{a}^{b} f(x) d x \int_{a}^{b} g(x) d x$, Math. Z., 39 (1935), 215-226. 3

[13] D. S. Mitrinović, J. E. Pecarić, A. M. Fink, Inequalities for Functions and Their Integrals and Derivatives, Kluwer Academic Publishers, Dordrecht, (1994). 1

[14] A. Ostrowski, Über die Absolutabweichung einer di erentienbaren Funktionen von ihren Integralimittelwert, Comment. Math. Helv., 10 (1938), 226-227. 1

[15] A. Qayyum, I. Faye, M. Shoaib, Improvement of Ostrowski Integral Type inequalities with application, Filomat (Accepted). 1

[16] A. Qayyum, S. Hussain, A new generalized Ostrowski Grüss type inequality and applications, Appl. Math. Lett., 25 (2012), 1875-1880. 1

[17] A. Qayyum, M. Shoaib, I. Faye, Some new generalized results on ostrowski type integral inequalities with application, J. Comput. Anal. Appl., 19 (2015), 693-712. 1

[18] A. Qayyum, M. Shoaib, I. Faye, On New Weighted Ostrowski Type inequalities Involving Integral Means over End Intervals and Application, Turk. J. Anal. Number Theory, 3 (2015), 61-67. 1

[19] A. Qayyum, M. Shoaib, A. E. Matouk, M. A. Latif, On New Generalized Ostrowski Type Integral inequalities, Abstr. Appl. Anal., 2014 (2014), 8 pages. 1, 2.3, 3.2, 4.5

[20] M. Z. Sarikaya, E. Set, On New Ostrowski Type Integral Inequalities, Thai J. Math., 12 (2014), 145-154. 1

[21] A. Sofo, S.S. Dragomir, An inequality of Ostrowski type for twice differentiable mappings in term of the $L_{p}-$ norm and applications, Soochow J. Math., 27 (2001), 97-111. 1 